\title{
An empirical analysis of real activity and stock returns in an emerging market
}

\begin{abstract}
The present paper analyzes the role of stock market returns as a predictor of real output for a fast-growing emerging market, Malaysia. In the analysis, forecasting equations for 1-, 2-, 4-, and 8-quarter forecasting horizons based on autoregressive distributed lags framework are adopted. From the estimation, we find evidence that stock market returns do contain predictive ability at short-forecasting horizons, especially at less than 4-quarter horizons. Estimating the forecasting models recursively, we note reduction of out-of-sample forecasting evaluation statistics, namely the mean absolute errors (MAE) and the mean squared forecast errors (MSFE), from those obtained from the simple autoregressive (AR) model. More importantly, the null hypothesis of equal predictive accuracy between the model with stock returns as a predictor and the AR model is rejected for the 1-quarter and 2-quarter forecasting horizons by the McCrakenô (2007) out-of-sample-F statistics.
\end{abstract}

Keyword: Stock market returns; Forecast 